\title{
Óxido nítrico e o aumento do desempenho nas atividades físicas com a
}

\section{suplementação de origem vegetal}

\author{
Nitric oxide and performance increase in physical activities with plant origin supplementation \\ Óxido nítrico y mayor rendimiento en actividades físicas con suplementación de origen vegetal
}

Recebido: 15/09/2021 | Revisado: 19/09/2021 | Aceito: 19/09/2021 | Publicado: 21/09/2021

\author{
Adriana Freitas dos Anjos \\ ORCID: https://orcid.org/0000-0002-2645-7928 \\ Centro Universitário Fametro, Brasil \\ E-mail: adriaanjo7@gmail.com \\ Antônia Luciane da Silva Peres \\ ORCID: https://orcid.org/0000-0002-3137-5177 \\ Centro Universitário Fametro, Brasil \\ E-mail: lucianeperes0@gmail.com \\ Caroline Cristina dos Santos Alcântara \\ ORCID: https://orcid.org/0000-0003-4286-0431 \\ Centro Universitário Fametro, Brasil \\ E-mail: caroline.alcantara@hotmail.com.br \\ José Carlos de Sales Ferreira \\ ORCID: https://orcid.org/0000-0002-1867-8229 \\ Centro Universitário Fametro, Brasil \\ E-mail: jcarlos.sales@gmail.com
}

\begin{abstract}
Resumo
Introdução: O desempenho físico demandado do substrato do óxido nítrico é favorecido com o aumento do aporte de oxigênio ao tecido muscular por meio da broncodilatação e da vasodilatação ocasionada pela ação metabólica do NO. Objetivo Geral: E como principal objetivo deste artigo está a compreensão do processo metabólico do óxido nítrico na fisiologia humana abrangendo a origem pela qual essa substância é desenvolvida ou adquirida no corpo humano através do consumo de suplementação de estirpe vegetal gerando os benefícios necessários para um bom desempenho nas atividades físicas. Metodologia: Como metodologia dos parâmetros conclusivos deste artigo, utilizou-se um levantamento bibliográfico da literatura científica. Resultado e Discussão: Os resultados esperados eram comprovar que é possível obter ótimos resultados no desempenho funcional em relação as atividades físicas no modo geral, através do consumo de alimentos ricos em nitrato. Conclusão: De fato, o Oxido Nítrico (NO), tem suma importância na fisiologia do exercício, pois, é uma substância que promove relaxamento dos vasos sanguíneos e regulação de pressão sanguínea, resultando em um maior fluxo de sangue, de oxigênio e de nutrientes por todo corpo.
\end{abstract}

Palavras-chave Óxido nítrico; Nitrato; Desempenho funcional; Atividade física; Suplementação.

\begin{abstract}
Introduction: The physical performance demanded of the nitric oxide substrate is favored with the increase in the oxygen supply to the muscle tissue through bronchodilation and vasodilation caused by the metabolic action of NO. General Objective: The main objective of this article is the understanding of the metabolic process of nitric oxide in human physiology, covering the origin by which this substance is developed or acquired in the human body through the consumption of vegetable strain supplementation, generating the benefits for good performance in physical activities. Methodology: As a methodology of the conclusive parameters of this article, a bibliographic survey of the scientific literature was used. Results and Discussion: The expected results were to prove that it is possible to obtain great results in the functional performance in relation to physical activities in general, through the consumption of foods rich in nitrate. Conclusion: In fact, Nitric Oxide (NO) is extremely important in exercise physiology, as it is a substance that promotes relaxation of blood vessels and regulation of blood pressure, impossible in a greater flow of blood, oxygen and nutrients all over the body.
\end{abstract}

Keywords: Nitric oxide; Nitrate; Functional performance; Physical activity; Supplementation.

\section{Resumen}

Introducción: El rendimiento físico exigido al sustrato de óxido nítrico se ve favorecido con el aumento del aporte de oxígeno al tejido muscular a través de la broncodilatación y vasodilatación provocada por la acción metabólica del NO. Objetivo General: Y el objetivo principal de este artículo es la comprensión del proceso metabólico del óxido nítrico en la fisiología humana, abarcando el origen por el cual esta sustancia se desarrolla o adquiere en el cuerpo humano a través del consumo de suplementos de cepas vegetales, generando la necesaria Beneficios para un buen 
desempeño en actividades físicas. Metodología: Como metodología de los parámetros concluyentes de este artículo se utilizó un relevamiento bibliográfico de la literatura científica. Resultados y Discusión: Los resultados esperados fueron demostrar que es posible obtener grandes resultados en el desempeño funcional en relación a las actividades físicas en general, a través del consumo de alimentos ricos en nitratos. Conclusión: De hecho, el Óxido Nítrico (NO) es de suma importancia en la fisiología del ejercicio, ya que es una sustancia que promueve la relajación de los vasos sanguíneos y la regulación de la presión arterial, lo que resulta en un mayor flujo de sangre, oxígeno y nutrientes por todo el cuerpo.

Palabras clave: Óxido nítrico; Nitrato; Presentación funcional; Actividad física; Suplementacion.

\section{Introdução}

A sociedade está cada vez mais inteirada na busca da prática de uma vida saudável, como, atividades físicas, alimentação funcional e suplementação alimentar para um bom desempenho físico, de tal maneira que, o próprio mercado alimentício, farmácias e lojas especializadas em mantimentos nutricionais vêm investindo massivamente nesta sessão, de modo que, os suplementos energéticos, proteicos ou de vitaminas sintéticas vem sendo alvo principal dos consumidores (Bacurau, 2009).

Segundo a Resolução do Conselho Federal de Nutrição (CFN) nº 380/2005, Suplementos Nutricionais sintéticos são alimentos que servem para complementar, com calorias, e ou nutrientes a dieta diária de uma pessoa saudável, em casos onde sua ingestão, a partir da alimentação, seja insuficiente, ou quando a dieta requerer suplementação. A mesma resolução também diz que eles devem conter um mínimo de $25 \%$ e no máximo $100 \%$ da ingestão diária recomendada (IDR) de vitaminas e/ou minerais, na porção diária indicada pelo fabricante, não podendo substituir os alimentos, nem serem considerados como dieta exclusiva.

Faz-se necessária uma orientação dietética adequada, antes da indicação de qualquer suplementação. A ingestão da quantidade correta de calorias, carboidratos, proteínas, gorduras, vitaminas e minerais é a base fundamental para qualquer praticante de atividade física. Para isso, a ISSN (International Society of Sports and Nutrition) publica recomendações periódicas sobre a quantidade adequada dos macros e micronutrientes na alimentação, que deve ser individualizada, dependendo da carga de atividade física de cada indivíduo (Costa \& Borba, 2015).

Conforme ressalta Moreira At. Al (2014), uma orientação nutricional correta e individualizada, promove a manutenção da saúde do atleta, além de favorecer o funcionamento das vias metabólicas associadas ao exercício físico, como por exemplo, a vasodilatação e o armazenamento de energia através da formação do glicogênio muscular como aliada na diminuição da fadiga muscular.

O Óxido Nítrico (NO), tem suma importância na fisiologia do exercício, onde, a sua principal matéria prima é o nitrato, e esta pode ser encontrada em alguns alimentos e vegetais, como, a beterraba juntamente com o espinafre, chocolate amargo, melancia sementes e nozes. O NO é uma substância que promove relaxamento dos vasos sanguíneos e regulação de pressão sanguínea, resultando em um maior fluxo de sangue, de oxigênio e de nutrientes por todo corpo. Além disso este demonstrou ter uma gama de outros efeitos vasculares benéficos, incluindo inibir a agregação plaquetária, preservar ou melhorar a disfunção endotelial, melhorar o desempenho no exercício em indivíduos saudáveis e pacientes com doenças arteriais periférica (Guiarone et al., 2014).

\section{Metodologia}

O objetivo da pesquisa foi de caráter descritivo, propondo a identificação, registro e análise das características, fatores ou variáveis que se relacionam com o fenômeno ou processo (Perovano, 2014). Dessa forma, embasada em referências bibliográficas e científicas, relatando Óxido nítrico e o aumento do desempenho nas atividades físicas com a suplementação de origem vegetal. 
Utilizou-se de um levantamento bibliográfico da literatura científica disponível em bibliotecas virtuais e sites, como as bases de dados PUBMED, LILACS, Google Scholar e SCIELO. Tomou-se como base as seguintes etapas: definição do tema, busca e seleção dos artigos, seleção das informações a serem extraídas, interpretação dos resultados e, por fim, síntese do conhecimento.

Foram encontrados 45 artigos nacionais e internacionais, dos quais foram utilizados 22 artigos para a elaboração do Trabalho de Conclusão de Curso (TCC), pois eram os que mais se aproximavam do objetivo do trabalho. Foi utilizada como critério de seleção a escolha de artigos que tivessem como foco os efeitos benéficos da suplementação natural para as práticas de atividades físicas.

Foram analisados artigos acadêmicos, publicações de revistas acadêmicas, diretrizes, livros, utilizados como forma de estudo, de pesquisa, de revisão integrativa para um projeto conciso mais completo.

\section{Resultados e Discussão}

\subsection{Metabolismo do óxido nítrico no corpo humano}

Define-se óxido nítrico como um gás solúvel, altamente lipofílico sintetizado pelas células endoteliais, macrófagos e certo grupo de neurônios do cérebro, e de acordo com Filho e Zilberstein (2000) o óxido nítrico é um importante neurotransmissor com capacidade potencializadora, atuando na memória e no aprendizado, podendo também ter ações endócrinas, autócrinas e parácrinas. A sua ação na imuno-regulação está presente na inflamação e nos mecanismos de autoimunidade. Esta molécula tem revolucionado e obrigado revisão de paradigmas da medicina, principalmente em neurologia, cardiologia, nefrologia e gastroenterologia.

O óxido nítrico (NO) constitui uma das menores e mais simples moléculas biossintetizadas. O NO é um radical livre, gasoso, inorgânico, incolor, que possui sete elétrons do nitrogênio e oito do oxigênio, tendo um elétron desemparelhado. A síntese do NO resulta da oxidação de um dos dois nitrogênios guanidino da L-arginina, que é convertida em L-citrulina. Esta reação é catalisada pela enzima NO-sintase (Figura 1). O NO produzido pelas células endoteliais tem um papel essencial no processo de relaxamento do vaso sanguíneo. Em condições fisiológicas, o relaxamento vascular ocorre quando receptores da membrana das células endoteliais são ativados por estímulos solúveis (incluindo-se acetilcolina, bradicinina, adenosina difosfato, substância $\mathrm{P}$, serotonina e outros) ou quando há um aumento do atrito exercido pelas células circulantes sobre a camada endotelial (shear-stress), levando à ativação da e-NOS presentes nestas células e à consequente produção de NO. A e-NOS está estrategicamente ancorada à membrana da célula endotelial, o que favorece a presença de grandes quantidades de NO próximo à camada muscular do vaso e às células sanguíneas circulantes. Em resposta a agonistas como a bradicina, ocorre a fosforilação da eNOS, determinando sua translocação para o citosol. Este mecanismo provavelmente tem um papel na regulação da produção de NO in situ e na sua atividade biológica. O NO produzido na célula endotelial difunde-se rapidamente para a célula muscular e para o lúmen vascular. A difusão rápida e a facilidade com que esta molécula penetra em outras células, graças ao seu pequeno tamanho e à sua característica lipofílica, são cruciais para o entendimento das suas atividades biológicas. No interior da célula muscular, o NO interage com o ferro do grupo heme da enzima guanilato ciclase, acarretando uma alteração da conformação desta enzima, tornando-a ativa (Dusse et al, 2003). 
Figura 1 - Reação catalisada pela NO-sintase.

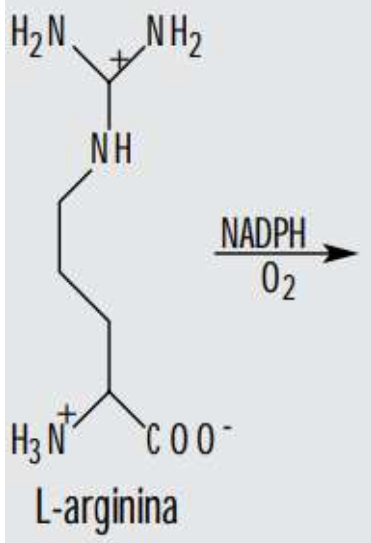

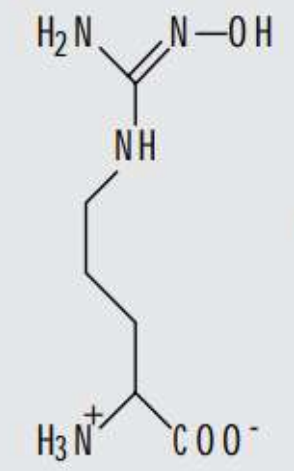

N-hidroxi-L-arginina

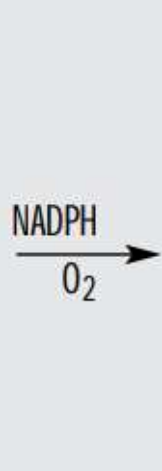

(1)

Fonte: Dusse et al (2003).

O óxido nítrico como regulador da pressão sanguínea, o controle da pressão arterial é uma das mais complexas funções fisiológicas, envolvendo os sistemas cardiovascular, renal, neural e endócrino. Sabe-se que no corpo humano existem 3 tipos de músculos: músculo estriado esquelético, músculo estriado cardíaco e músculo liso. Os músculos lisos são involuntários e encontram-se envolvendo a parede de órgãos ocos. São responsáveis, dentre outros fenômenos, pelas contrações que empurram os alimentos ao longo do tubo digestivo e que diminuem o calibre das artérias, aumentando a pressão do sangue. Murad e colaboradores sugeriram, no início dos anos oitenta, que a relaxação no músculo liso requer a ativação da enzima guanilato ciclase e seria acompanhada pela conversão da guanosina trifosfato (GTP) em guanosina monofosfato cíclico (cGMP), sendo este processo de relaxação desencadeado por mensageiros químicos, tal como a acetilcolina, que atuariam diretamente nas células musculares do sistema vascular. Porém, em 1980, Furchgott e Zawadzki descobriram que, na realidade, os mensageiros químicos responsáveis pela dilatação dos vasos sanguíneos agem na camada celular, chamada endotélio, que reveste o interior do vaso sanguíneo. Em reação ao estímulo do mensageiro químico, o endotélio produz uma molécula mensageira que se difunde pelas células musculares situadas sobre ele, que em seguida se relaxam. Furchott e Zawadzki chamaram essa misteriosa molécula de fator relaxante derivado do endotélio de acordo com EDRF (1980) citado por Queiroz et al, (1999).

\subsection{Fontes naturais de óxido nítrico}

Segundo Close et al (2016) nos últimos anos, foram identificados diferentes macronutrientes e micronutrientes com capacidade de melhorar o desempenho esportivo, e um deles é o foco principal, o óxido nítrico (NO), o qual possui um efeito vasodilatador, apresentando uma grande importância hemodinâmica, por melhorar o fluxo sanguíneo e a oxigenação muscular, além de reduzir a pressão arterial (Erzurum et al, 2007). Do mesmo modo, redução da utilização de O2, fosfocreatina e custo por ATP (Bailey et al, 2010), estímulo da expressão gênica de biogênese mitocondrial e aumento de sua eficiência (Larsen et $a l, 2011)$, captação de glicose no músculo e aumento da conversão de fibras tipo II estão entre os efeitos que a ingestão dessa substância podem trazer ao organismo (Figura 2). 
Figura 2 - Vias de produção do NO.

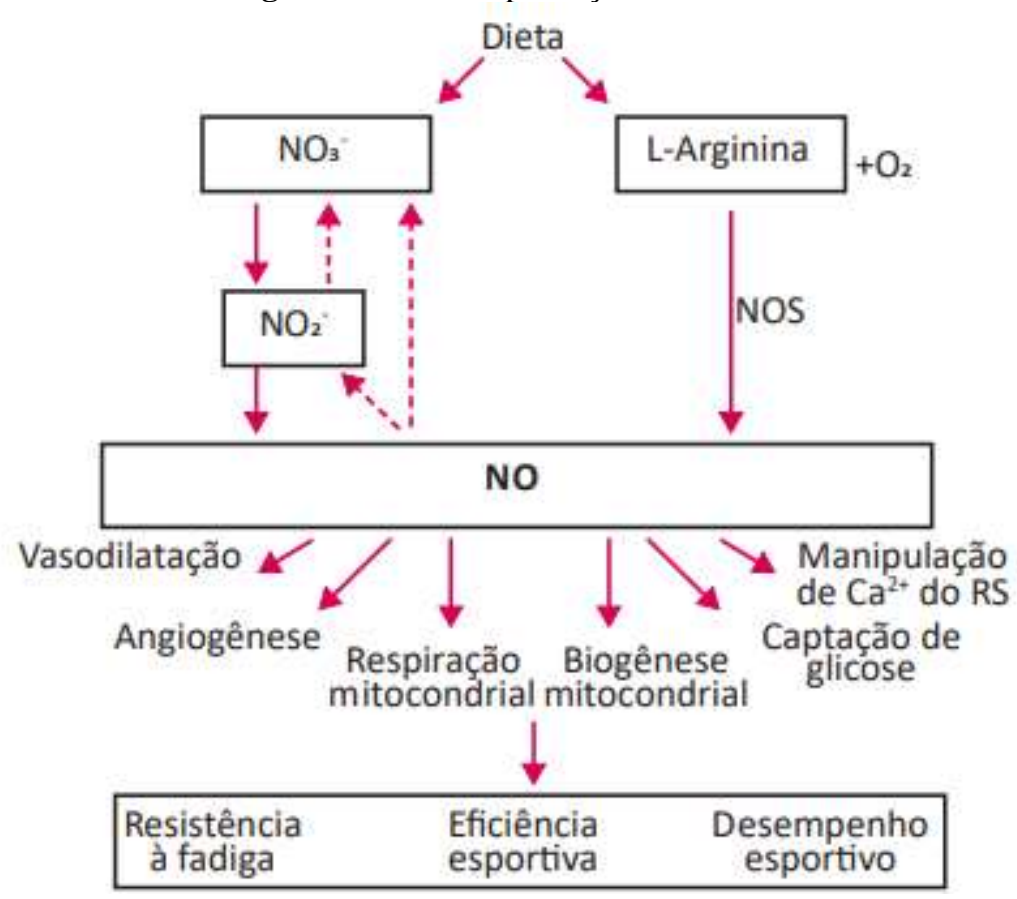

Fonte: Traduzido por Jones (2014).

O NO é produzido a partir de L-arginina e oxigênio em reação catalisada pelas NO sintases e é, subsequentemente, oxidado a nitrito e nitrato. O nitrato pode ser reduzido a nitrito, e o nitrito pode ser, ainda, reduzido a NO, um efeito que é acentuado quando a disponibilidade de oxigênio no tecido é baixa. Desta forma, os produtos de produção de NO podem ser reciclados. Além da produção endógena, as reservas corporais de nitrato e nitrito podem ser aumentadas por meio do consumo de alimentos ricos em nitrato inorgânico. O NO é importante em vários processos fisiológicos que podem melhorar o desempenho esportivo. É possível que a dependência da via nitrato-nitrito-NO para a produção de NO seja aumentada durante o exercício. As setas tracejadas mostram que o NO pode ser oxidado para NO2 - e NO3 -. Ca2+ Cálcio, NO óxido nítrico, NO3 - nitrato, NO2 - nitrito, NOS óxido nítrico sintase, RS retículo sarcoplasmático (JONES, 2014).

Segundo Hord et al (2009) o nitrato inorgânico pode ser encontrado em uma variedade de vegetais e frutas. Quando ingerido, uma parte de NO3 é reduzido a NO2 - por bactérias comensais anaeróbias facultativas presentes na cavidade oral, por ação do nitrato redutase (Figura 2) e, então, convertido em óxido nítrico no estômago (Lundberg \& Govoni, 2004). Para este processo os níveis de vitamina $\mathrm{C}$ e polifenóis devem ser em quantidade adequadas, uma vez que contribuem neste processo de conversão. Entretanto, uma quantidade substancial de nitrito não é convertida e entra na circulação sistêmica, elevando a concentração plasmática após 2-3 horas, e a concentração plasmática de nitrato possui seu pico sanguíneo entre 1-2 h após a ingestão em bolus (Webb et al, 2008). Uma variedade de enzimas, proteínas e nutrientes, incluindo xantina oxidase, desoxihemoglobina, ascorbato e polifenóis, podem catalisar a redução de um elétron do nitrito para NO no sangue e em outros tecidos (Shiva et al, 2007).

O nitrato inorgânico ingerido de fontes dietéticas é rapidamente absorvido no intestino delgado. Embora grande parte do nitrato circulante seja eventualmente excretada na urina, até $25 \%$ é ativamente extraída pelas glândulas salivares e concentrada na saliva. Na boca, as bactérias anaeróbias comensais facultativas reduzem efetivamente nitrato a nitrito pela ação de enzimas nitrato redutase. No estômago, em meio ácido, o nitrito é espontaneamente decomposto para formar óxido nítrico (NO) e outros óxidos de nitrogênio bioativos, que regulam importantes funções fisiológicas. Nitrato e nitrito restante é 
absorvido a partir do intestino para a circulação e pode converter para NO no sangue e tecidos sob hipóxia fisiológica (Lundberg et al, 2008).

Figura 3 - A circulação entero-salivar do nitrato em seres humanos.

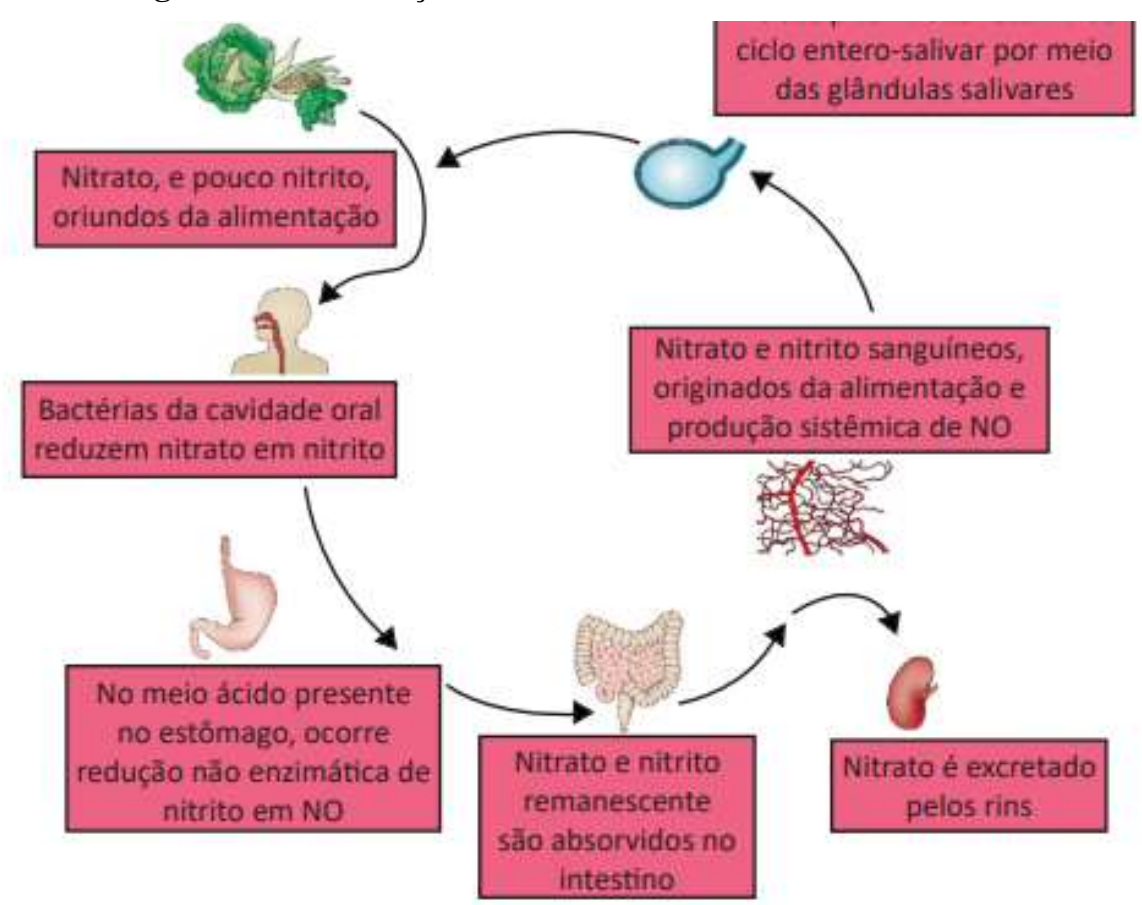

Fonte: Traduzido de Lundberg et al (2008).

Os vegetais são as principais fontes dietéticas de nitrato, sendo destacados pelo alto teor dessa substância (> 1000 mg/kg), sobressaindo-se o espinafre, a alface e a beterraba (Parizotti, 2008; Govoni, 2004; Tamme et al., 2004).

De acordo com Parizotti (2008) citado por Paula at al (2017) a redução da pressão arterial pelo uso do suco de beterraba é relacionada ao seu conteúdo de nitrato, o qual, ao ser convertido em nitrito, será capaz de aumentar a biodisponibilidade de NO, composto conhecido por seu efeito de vasodilatação por meio do efeito de relaxamento do músculo liso, dado pela síntese de guanosinamonosfofato cíclica a partir da guanosina trifosfato. A beterraba também está sendo considerada como promissora no tratamento terapêutico de uma gama de patologias clínicas associadas com stress oxidativo e inflamação (Clifford et al., 2015). O nitrato, ingerido via beterraba crua, recentemente ganhou atenção considerável como um meio alimentar para o aumento da biodisponibilidade do óxido nítrico (Bailey et al., 2009).

As fontes alimentares de nitrato são classificadas conforme o teor de nitrato por $100 \mathrm{~g}$ de alimento (Tabela 1). Apesar de a avaliação da quantidade de nitrato ser em mg, os estudos trabalham com doses em mmol. A conversão é feita por uma constante, sendo 0,1 mmol para cada 6,4 mg. Além do seu teor, alimentos fonte de nitrato inorgânico deve ser escolhidos conforme sua biodisponibilidade. Van Velzen et al, 2008, compararam a biodisponibilidade entre nitrato de sódio, espinafre, alface e beterraba e concluíram que a alface possuiu o dobro de biodisponibilidade que o espinafre, além de todos os alimentos serem superiores ao nitrato de sódio. 
Tabela 1 - Classificação dos alimentos quanto ao conteúdo de nitrato em $100 \mathrm{~g}$ de alimento fresco.

\begin{tabular}{ll}
\hline $\begin{array}{c}\text { Conteúdo de nitrato } \\
\text { (mg) em } 100 \mathrm{~g} \text { de } \\
\text { alimento fresco }\end{array}$ & \multicolumn{1}{c}{ Alimentos } \\
\hline Muito baixo <20 mg & $\begin{array}{l}\text { Aspargo, cebola, feijão, cogumelos, ervilha, pimenta, } \\
\text { tomate, melancia e alho } \\
\text { Brócolis, cenoura, couve-flor, pepino, abóbora e } \\
\text { chicória } \\
\text { Repolho, endro e nabo } \\
\text { Bvelã, repolho chinês, endívia, funcho, alho-poró, } \\
\text { salsinha e couve-rábano }\end{array}$ \\
Moderado $<100 \mathrm{mg}$ & $\begin{array}{l}\text { Aipo, agrião, cerefólio, alface, beterraba, espinafre e } \\
\text { rúcula }\end{array}$ \\
\hline Muito alto $<250 \mathrm{mg}$ & $\mathrm{mg}$ \\
\hline
\end{tabular}

Fonte: Traduzido e adaptado de: Hord, Tang e Bryan (2011).

\subsection{Os benefícios do óxido nítrico ao bom desempenho físico}

Loureiro e Santos (2017), afirmam que o óxido nítrico possui papel importante na regulação de parâmetros funcionais e fisiológicos relacionados ao desempenho esportivo. Recentemente, foi comprovada sua ação vasodilatadora e hipotensiva, bem como no aumento da eficiência energética e na melhora da contração muscular além de conversão de fibras musculares. Sua principal via de formação é dependente das óxido nítrico sintases, enzimas dependentes de oxigênio que, em hipóxia, reduzem sua ação. Nestas situações, o NO é formado a partir do nitrato inorgânico (NO3) e nitrito provenientes da alimentação. O aumento da ingestão de NO3, por alimentação ou suplementação, parece promover aumento de desempenho esportivos em diversos tipos de exercício. Entretanto, seu efeito ergogênico é dependente do condicionamento físico, tipo e duração do exercício e da dose administrada. Indivíduos com menor condicionamento físico parecem se beneficiar mais dos efeitos da suplementação que indivíduos treinados. Além disso, a suplementação parece potencializar o desempenho de forma mais consistente frente a exercícios de alta intensidade e curta duração quando comparada com seus efeitos sobre exercícios de endurance.

Segundo Nicastro (2016) o metabolismo do nitrato em nosso organismo é favorável, uma vez que pouco se perde pelo trato gastrointestinal e o restante é absorvido, elevando os níveis sanguíneos de nitrato para a formação de óxido nítrico (NO), gerando a resposta de vasodilatação. A beterraba, conhecida por ser fonte de antioxidantes, potássio, sódio, betaína, magnésio e vitamina $\mathrm{C}$ é também uma das fontes alimentares mais ricas em nitrato. O consumo de suco de beterraba concentrado (o industrializado feito com matéria-prima natural, não o suco convencional caseiro) tem demonstrado ser extremamente eficiente em reduzir o custo energético para determinada tarefa muscular. Por exemplo, ingerir suco de beterraba concentrado antes do exercício aeróbio reduz o custo de oxigênio e aumenta a tolerância ao esforço. Já no exercício de força de alta intensidade, reduz o custo de energia para que o músculo desempenhe contrações de força máxima com mais eficiência.

Em termos práticos, o consumo de nitrato, por meio do suco de beterraba concentrado, proporciona aumento na tolerância ao esforço, que se traduz em maior número de repetições executadas até a falha muscular e de carga suportada durante o exercício. Além disso, a ingestão do suco de beterraba concentrado pode reduzir a sensação de dor muscular tardia ocasionada pelo exercício de força de alta intensidade, dor essa que muitas vezes se reflete em maior perda de força muscular e queda de desempenho nas sessões seguintes de treino.

O consumo de nitrato per se não apresenta potencial de toxicidade. Seus metabólitos levantam a hipótese de possíveis ocorrências de efeitos adversos como carcinogênese, carcinoma renal e lesão renal aguda, quando consumido em altas dosagens ou de modo crônico. Há evidências recentes, porém, demonstrando que o consumo de alta dosagem de sal de nitrato 
durante 7 dias consecutivos não causou qualquer efeito deletério sobre a função renal de indivíduos saudáveis tanto em repouso quanto após o exercício de alta intensidade. Portanto, se a suplementação de nitrato realizada em alta dosagem e cronicamente na forma de sal não induz qualquer efeito deletério na função renal, a forma concentrada do suco de beterraba (desde que de origem natural, é segura).

\subsection{Recursos ergogênicos encontrados em alimentos}

A ergonomia estuda a relação entre o homem e seu ambiente de trabalho. Nesse sentido, o termo ambiente não se refere apenas ao contorno ambiental, no qual o homem desenvolve suas atividades, mas também as suas ferramentas, seus métodos de trabalho e à organização deste levando-se em consideração este homem tanto como indivíduo quanto como participante de um grupo de trabalho (Ferreira, 2008).

O profissional nutricionista atua como um promotor da saúde, ajudando na qualidade de vida dos colaboradores, identificando e adequando o ambiente de trabalho, evitando assim, o absenteísmo. A ergonomia não está somente preocupada com as condições físicas do trabalho, mas também, com sua organização. A ergonomia busca examinar o conteúdo das tarefas, os ritmos impostos aos trabalhadores, a divisão do trabalho, as relações de poder, as relações interpessoais, fatores estes que convergem para a desmotivação e insatisfação dos trabalhadores, no exercício de suas atividades (Marcon, 1997).

A partir do final da primeira década deste século, surgiu uma linha de pesquisa com número de publicações crescentes ao longo dos anos, mostrando que diversos alimentos têm potencial ergogênico, por melhorar o desempenho de atletas recreacionais e de alto nível, diminuir as atividades oxidantes e inflamatórias induzidas pelo exercício físico (Yavari et al., 2015).

Estudos têm mostrado melhorias da capacidade aeróbica e redução da produção de lactato fornecidos pelo suco de laranja e hortelã-pimenta. Onde o consumo crônico durante três meses de $500 \mathrm{~mL} / \mathrm{d}$ de suco de laranja mostrou ser eficaz em reduzir a concentração de lactato e melhorar o desempenho em testes realizados após treinamento aeróbico por uma hora, três vezes por semana, em mulheres (Aptekmann \& Cesar, 2010). Para Meamarbashi e Rajabi (2013), a ingestão de óleo essencial de hortelã-pimenta $(0,05 \mathrm{~mL})$ em $500 \mathrm{~mL}$ de água mineral por dez dias resultou em redução na concentração de lactato e melhora no desempenho em teste até a exaustão realizado após a suplementação em jovens saudáveis.

A ingestão de vitamina $C$ teve aumento significativo para as mulheres eutróficas e com sobrepeso, embora o aumento para as últimas não tenha sido significante. Para os homens, a vitamina $\mathrm{C}$ aumentou significativamente nos eutróficos e com sobrepeso, atingindo a recomendação.

Tabela 2 - Variáveis antropométricas em mulheres $(n=15)$ e homens $(n=14)$ distribuídas de acordo com o índice de massa corporal (IMC) no início do experimento (pré) e ao final (pós) da suplementação com suco de laranja.

\begin{tabular}{|c|c|c|c|c|c|c|c|c|c|c|c|c|c|c|c|c|}
\hline \multirow{4}{*}{ Suco laranja } & \multicolumn{8}{|c|}{ Mulheres } & \multicolumn{8}{|c|}{ Hamens } \\
\hline & \multicolumn{4}{|c|}{$\begin{array}{l}\text { Eutrotia }\left\{\leq 25 \mathrm{~kg} / \mathrm{m}^{2}\right\} \\
\qquad\{\mathrm{n}=10)\end{array}$} & \multicolumn{4}{|c|}{$\begin{array}{c}\text { Sobrobeso }\left(-25 \mathrm{~kg} / \mathrm{m}^{2}\right\rangle \\
\langle(n=5\}\end{array}$} & \multicolumn{4}{|c|}{$\begin{array}{l}\text { Eutrofia }(\leq 25 \mathrm{~kg} / \mathrm{m}) \\
\qquad(\mathrm{n}=9)\end{array}$} & \multicolumn{4}{|c|}{$\begin{array}{c}\text { Sobuobeso }\left(>25 \mathrm{~kg} / \mathrm{m}^{\prime}\right) \\
(n=5)\end{array}$} \\
\hline & \multicolumn{2}{|c|}{ Pre } & \multicolumn{2}{|c|}{$P B O S$} & \multicolumn{2}{|c|}{ Pré } & \multicolumn{2}{|c|}{ Pos } & \multicolumn{2}{|c|}{ lire } & \multicolumn{2}{|c|}{ ios } & \multicolumn{2}{|c|}{ Pres } & \multicolumn{2}{|c|}{ Pos } \\
\hline & $M$ & $\overline{D P^{2}}$ & $M$ & Dip & $M$ & $\overline{\mathrm{DP}}$ & $M$ & $\overline{D P}$ & $M$ & $\overline{\mathrm{DI}} \mathrm{P}^{2}$ & $M$ & DP & $M$ & $\overline{\text { DP }}$ & $M$ & 멀 \\
\hline Reso (kg) & 58 & 7 & 57 & 7 & 70 & 7 & 70 & 5 & 65 & 5 & 67 & 5 & 93 & 16 & 93 & 16 \\
\hline IMC $(\mathrm{kg} / \mathrm{m} /)$ & 21 & 2 & 21 & 2 & 28 & 3 & 28 & 3 & 22 & 1 & 22 & 1 & 30 & 3 & 30 & 3 \\
\hline Gorcura corporal (itu) & 28 & 4 & 26 & 4 & 34 & 4 & 34 & 3 & 22 & 6 & 21 & 3 & 27 & 5 & 21 & 6 \\
\hline Circunfertencia abocominal (cm) & 77 & 6 & 77 & 5 & 90 & 7 & so & 7 & 79 & 7 & 79 & 7 & 102 & 13 & 104 & 12 \\
\hline
\end{tabular}

Fonte: Rev. Nutr., Campinas, 23(5): 779-789. (2010) 
Além do suco de laranja, tem-se também, o suco de beterraba, que pode ser consumido antes da prática de exercício físico, reduzindo a pressão arterial sistêmica, o consumo de oxigênio, a produção e liberação de lactato sanguíneo, aumentando o rendimento e melhorando o desempenho do exercício físico. Ciclistas trinados, fez- se a suplementação com o suco de beterraba por um período de seis dias, diminuiu o volume de oxigênio durante o exercício aumentando então o desempenho em uma prova de $10 \mathrm{~km}$. O grupo suplementado com o suco rico em NO teve i desempenho em provas de 4 e 16,1 km, em decorrência de uma melhora significativa na potência para os mesmos valores de VO2. (Cermal, Gibala \& Van Loon, 2012).

\section{Conclusão}

Mediante os resultados obtidos, conclui-se que, o óxido nítrico utilizado como forma suplementar e de origem vegetal na alimentação nos pré-treinos diariamente, tem contribuído de forma benéfica para o desempenho funcional. Um exemplo de fonte de nitrato, a principal matéria prima do NO, é a beterraba, que apesar de poucos estudos evidenciados em artigos científicos, tem função vasodilatadora, aumentando a introdução de oxigênio muscular, aumentando a oferta de glicose e micronutrientes como; ácido ascórbico, sódio, potássio e magnésio. e a redução da pressão sanguínea. Incluído na dieta de indivíduos ativos, como esportistas, onde este auxilia na performance de atividades físicas, reduzindo a perturbação metabólica do musculo durante o treino e a redução da pressão sanguínea. O consumo da beterraba e outros vegetais devem ser incentivados na dieta, não só pelo seu teor de nitrato, mas também pela oferta de vitaminas, fibras e minerais essenciais para saúde do corpo.

\section{Referências}

Bacurau, R. F. (2009). Nutrição e suplementação esportiva. Phorte. (6a ed.).

Bailey, J. S. et al. (2009). Dietary nitrate supplementation reduces the $\mathrm{O} 2$ cost of low-intensity exercise and enhances tolerance to high-intensity exercise in humans. Journal Appl. Physiology, 107(4), 1144-1155. https://pubmed.ncbi.nlm.nih.gov/19661447/.

Bailey, S. J., Fulford, J., Vanhatalo, A., et al. (2010). Dietary nitrate supplementation enhances muscle contractile efficiency during kneeextensor exercise in humans. J Appl Physiol; 109, 135-48.

Bleil, S. I. (1998). O Padrão Alimentar Ocidental: Considerações Sobre a Mudança de Hábitos no Brasil. Cadernos de Debate. 6, 1-25.

Brum, P. C., Forjaz, C. L. M., Tinucci, T., \& Negrão, C. E. (2004). Adaptações agudas e crônicas do exercício físico no sistema cardiovascular. Revista Paulista de Educação Física, 18, 21-31.

Cervato, A. M., Mazzilli, R. N., Martins, I. S., \& Marucci, M. F. N. (1997). Dieta habitual e fatores de risco para doenças cardiovasculares. Rev. Saúde Pública, 31 (3), 227-35.

CFN. Resolução CFN N. 380/2005. Dispõe sobre a definição das áreas de atuação do nutricionista e suas atribuições, estabelece parâmetros numéricos de referência, por área de atuação e dá outras providencias. Num. 380 de 9 de dezembro de 2005. Brasília.

Clifford, T. et al. The potential benefits of red beetroot supplementation in health and disease. Journal Nutrients, 7, 2801-2822, https://www.ncbi.nlm.nih.gov/pmc/articles/PMC4425174/pdf/nutrients-07-02801.pdf.

Close, G.L., Hamilton, D.L., Philp, A., et al. New strategies in sport nutrition to increase exercise performance. Free Radic Biol Med; 98 : 144-58, 2016.

Costa, T. M. R. L., \& Borba, V. Z. C. (2015). Suplementos nutricionais. Nutritional Supllements. Revista médica da UFPR. Rev. Med. UFPR 2(3), 123-133.

Danson, E. J., \& Paterson, D. J. (2005). Cardiac neurobiology of nitric oxide synthaes. Annals New York Academy of Sciences, 1047(1), 183-196

Dusse, L. M. S., VIEIRA, L. M., \& Carvalho, M. G. (2003). Revisão sobre óxido nítrico. Jornal Brasileiro de Patologia e Medicina Laboratorial. 39(4), 343350 .

Erzurum, S. C., Ghosh, S., Janocha, A. J., et al. (2007). Higher blood flow and circulating NO products offset high-altitude hypoxia among Tibetans. Proc Natl Acad Sci; 104, 17593-8.

Filho, R. F., \& Zilberstein, B. (2000). Óxido nítrico: o simples mensageiro percorrendo a complexidade. Metabolismo, síntese e funções. Departamento de Gastroenterologia da Faculdade de Medicina da Universidade de São Paulo, São Paulo, SP. Rev. Assoc. Med. Bras..46(3) 
Gago, B., Lundberg, J. O., Barbosa, R. M. et al. (2007). Red wine-dependent reduction of nitrite to nitric oxide in the stomach. Free Radic Biol Med; 43: 123342.

Garcia, R. W. D. (2003). Reflexos da globalização na cultura alimentar: considerações sobre as mudanças na alimentação urbana. Revista de Nutrição, Campinas.

Ghiarone, T. E., Silva, T. A., Bertuzzi, R., Silva, A. E. L. (2014). Suplementação De Nitrato E Sua Relação Com A Formação De Óxido Nítrico E Exercício Físico. Artigo de revisão. Rev. Acta Brasileira do Movimento Humano. 4(4), 103 -135.

Green, D. J., Maiorana, A., O'driscoll G., Taylor, R. (2204). Effect of exercise training on endotheliumderived nitric oxide function in humans. The Journal of. Physiology, Cambridge, 561(1), 1-25.

Hord, N. G., Tang, Y., \& Bryan, N. S. (2009). Food sources of nitrates and nitrites: the physiologic context for potential health benefits. Am J Clin Nutr; 90 (1), $1-10$.

Irigoyen, M. C., Lacchini, S., Angelis, K., \& Michelini, L. (2003). Fisiopatologia da Hipertensão: O que avançamos? Revista da Sociedade de Cardiologia do Estado de São Paulo. São Paulo, 13(1), 20-45.

Irigoyen, M. C., Consolim-Colombo, F. M., \& Krieger, E. M. (2001). Controle cardiovascular: regulação reflexa e papel do sistema nervoso simpático. Revista Brasileira de Hipertensão, São Paulo, 8, 55-62.

Jones, A.M. (2014). Dietary Nitrate Supplementation and Exercise Performance. Sports Med; 44 (Suppl 1): S35-45.

Larsen, F. J., Schiffer, T. A., Borniquel, S., et al. (2011). Dietary inorganic nitrate improves mitochondrial efficiency in humans. Cell Metab; 13: 149-59.

Loureiro, L. L., \& Santos, G. B. (2021). Nitrato: suplementação, fontes dietéticas e efeitos na performance. Revista Brasileira de Nutrição Funcional. https://www.vponline.com.br/portal/noticia/pdf/24797f22647902947a312af063ec124d.pdf.

Lundberg, J. O., \& Govoni, M. (2004). Inorganic nitrate is a possible source for systemic generation of nitric oxide. Free Radic Biol Med; 37, 395-400.

Lundberg, J. O., Weitzberg, E., Gladwin, M. T. (2008). The nitrate-nitrite-nitric oxide pathway in physiology and therapeutics. Nat Rev Drug Discov; 7 (2): 156-67.

Maiorana, A., O’Driscoll, G., Dembo, L., Goodman, C., Taylor, R., \& Green, D. (2001). Exercise trainig, vascular function, and functional capacity in middleaged subjects. Medicine \& Science in Sport \& Exercise, Baltimore, 33(12), 2022-2028.

Martins-Pinge, M. C., Baraldi-Passy, I., \& Lopes, O. U. (1997). Excitatory effects of nitric oxide within the rostral ventrolateral medulla of freely moving rats. Hypertension, Dallas, 30, 704-707.

McARDLE, W., et al. (2003). Fisiologia do Exercício: Energia nutrição e Desempenho humano. (5a ed.). Guanabara Koogan.

Mckelvie, R. S., Teo, K. K., McCartney, N., et al. (1995). Efeitos do exercício físico em pacientes com insuficiência cardíaca congestiva: Uma revisão crítica. $J$ Am Coll Cardiol (ed. bras), 1, 300-8.

Monteiro, C. A., Mondini, L., \& Costa R. B. L. (2000). Mudanças na composição e adequação nutricional da dieta alimentar nas áreas metropolitanas do Brasil (1988-1996). Revista de Saúde Pública, 34(3), 251-58.

Moreira, F. P., \& Rodrigues, K. L. (2014). Conhecimento nutricional e suplementação alimentar por praticantes de exercícios físicos. Rev Bras Med Esporte, 20(5), São Paulo

Nair, S., Irving, B., \& Lanza, I. (2011). Can dietary nitrates enhance the efficiency of mitochondria? Cell Metabolism, 2(13), 117-118. https://www.ncbi.nlm.nih.gov/pmc/articles/PMC3049330/pdf/nihms268777.pdf>

Nahas, M. V. (2001). Atividade física, saúde e qualidade de vida: Conceitos e sugestões para um estilo de vida ativo. Miografi.

Nicastro, Humberto. (2016). Suco de beterraba e seus efeitos no exercício de força. https://blog.integralmedica.com.br/suco-de-beterraba-concentrado-nitratoe-seus-efeitos-no-exercicio-de-forca/.

Nisoli, E., Clementi, E., Paolucci, C., et al. (2003). Mitochondrial biogenesis in mammals: the role of endogenous nitric oxide. Science; 299, 896-99, 2003.

Parizotti, C. (2008). Suplementação com o suco de beterraba no exercício físico. In: Lundberg, J. O., Weitzberg, E., \& Gladwin, M. T. The nitrate-nitrite-nitric oxide pathway in physiology and therapeutics. Journal Nature Reviews Drug Discovery, 5(2), 156-167, https://pubmed.ncbi.nlm.nih.gov/18167491/.

Paula, A. M. S., Araújo, K. K. O., Santana, M. C. F. O., Bucioli, S. A., Melo, F. R. G., Verri, E. D. (2017). O uso da beterraba como vasodilatador em praticantes de atividades físicas. Ling. Acadêmica, Batatais, 7(5), 77-84.

Pereira, M. A., Tavares, M. R., \& Silva, R. B. V. (2014). Efeitos do suco de beterraba na performance de exercícios de endurance. RBNE - Revista Brasileira De Nutrição Esportiva, 8(47). Recuperado de http://www.rbne.com.br/index.php/rbne/article/view/471

Peri, L., Pietraforte, D., Scorza, G. et al. (2005). Apples increase nitric oxide production by human saliva at the acidic $\mathrm{pH}$ of the stomach: a new biological function for polyphenols with a catechol group? Free Radic Biol Med, 39: 668-81.

Perovano, D. G. (2014). Manual de Metodologia Científica. Editora Juruá.

Queiroz, F. L. N. (2008). Alimentação regional saudável em unidades produtoras de refeições do sudeste brasileiro. 2008.119 f. Dissertação (Mestrado em Nutrição Humana) -Universidade de Brasília, Brasília. 
Research, Society and Development, v. 10, n. 12, e289101220652, 2021

(CC BY 4.0) | ISSN 2525-3409 | DOI: http://dx.doi.org/10.33448/rsd-v10i12.20652

Queiroz, S. L., Batista, Alzir Azevedo. (1999). Funções biológicas do óxido nítrico. Quím. Nova, 22(4).

Santos, A. V., \& Farias, F. O. (2017). Consumo de suplementos nutricionais por praticantes de atividades físicas em duas academias de Salvador-BA. Revista Brasileira de Nutrição Esportiva, São Paulo. 11(64), 454-461.

Shiva, S., Huang. Z., Grubina, R., et al. (2007). Deoxymyoglobin is a nitrite reductase that generates nitric oxide and regulates mitochondrial respiration. Circ Res; 100: 654-61.

Souza, M. D. C. A., \& Hardt, P. P. (2002). Evolução dos hábitos alimentares no Brasil. Brasil alimentos.

Tamme, T. et al. (2004). Nitrates and nitrites vegetables and vegetables-based products and their intakes by estoniam population. Journal Food Additives and Contaminants, 23(4), 355-361. https://pubmed.ncbi.nlm.nih.gov/16546882/.

Van Velzen, A. G., Sips, A. J., Schothorst, R. C., Lambers, A. C. et al. (2008). The oral bioavailability of nitrate from nitrate-rich vegetables in humans. Toxicol Lett,181(3), 177-81.

Vanni, D. S., et al. (2007). Óxido nítrico, inibição das plaquetas e participação na formação do trombo. Jornal Brasileiro de Patologia e Medicina Laboratorial, 43(3), 181-189. http://www.scielo.br/pdf/jbpml/v43n3/a07v43n3.pdf.

Viaro, F., Nobre, F., \& Evora, P. R. B. (2000). Expressão das Óxido Nítrico Sintetases na Fisiopatologia das Doenças Cardiovasculares. http://publicacoes.cardiol.br/abc/2000/7404/74040009.pdf.

Webb, A. J., Patel, N., \& Loukogeorgakis, S. et al. (2008). Acute blood pressure lowering, vasoprotective, and antiplatelet properties of dietary nitrate via bioconversion to nitrite. Hypertension; 51, 784-90. 